\title{
Elevated Serum Complement Clq Levels After Traumatic Brain Injury and Its Association with Poor Prognosis
}

\author{
Xin-Jiang Yan' \\ Yang-Bo Li' \\ Wei Liu (D) \\ Hua-Yong $W^{2}$ \\ Guo-Feng $\mathrm{Yu}^{\prime}$ \\ 'Department of Neurosurgery, The \\ Quzhou Affiliated Hospital of Wenzhou \\ Medical University, Quzhou People's \\ Hospital, Quzhou, People's Republic of \\ China; ${ }^{2}$ Neurosurgical Intensive Care \\ Unit, The Quzhou Affiliated Hospital of \\ Wenzhou Medical University, Quzhou \\ People's Hospital, Quzhou, People's \\ Republic of China
}

\begin{abstract}
Objective: Complement $\mathrm{C} 1 \mathrm{q}$ is implicated in neuroinflammation. We intended to discern the relationship between serum $\mathrm{C} 1 \mathrm{q}$ levels and severity in addition to prognosis following traumatic brain injury (TBI).
\end{abstract}

Methods: In this prospective, observational study, serum C1q levels were quantified in 188 TBI patients and 188 healthy controls. Glasgow coma scale (GCS) and Rotterdam computed tomography (CT) classification were used as clinical and radiological indicators of severity. Patients with extended Glasgow outcome scale (GOSE) score of 1-4 at 6 months after trauma were considered to have a poor outcome. Multiple logistic regression model was built to ascertain the independent association of serum $\mathrm{C} 1 \mathrm{q}$ levels with 6-month poor outcome. Receiver operating characteristic (ROC) curve was configured for analysis of prognostic capability with respect to serum $\mathrm{C} 1 \mathrm{q}$ levels.

Results: TBI patients exhibited substantially higher serum C1q levels than controls (median, $223.9 \mathrm{mg} / 1$ versus $75.4 \mathrm{mg} / \mathrm{l}$ ). Serum C1q levels of patients were tightly correlated with GCS score $(r=-0.671)$, Rotterdam CT classification $(r=0.604)$ and GOSE score $(r=-0.581)$. An area under the ROC curve was yielded of 0.793 (95\% confidence interval $=0.728-0.849)$, and serum $\mathrm{C} 1 \mathrm{q}$ levels above $345.5 \mathrm{mg} / \mathrm{l}$ discriminated the risk of 6-month poor outcome with $59.6 \%$ sensitivity and $92.6 \%$ specificity. Serum C1q levels above $345.5 \mathrm{mg} / 1$ retained as an independent predictor for 6-month poor outcome with odds ratio of 4.922 (95\% confidence interval $=1.552-15.606 ; \mathrm{P}=0.017)$.

Conclusion: Elevated serum $\mathrm{C} 1 \mathrm{q}$ levels are closely correlated with traumatic severity and independently predicted the risk of long-term poor prognosis after TBI.

Keywords: traumatic brain injury, C1q, severity, functional outcome, biomarkers

\section{Introduction}

Traumatic brain injury (TBI) is one of the leading causes of death and disability globally. ${ }^{1}$ Inflammatory response is implicated in pathophysiological processes underlying secondary injury events after TBI. ${ }^{2}$ The complement system is an essential element in the inflammatory reaction, which is activated via three complement activation pathways, namely classical, alternative and lectin. ${ }^{3}$ The complement system contributes to neuronal cell death, brain edema, disruption of blood-brain barrier and infiltration of inflammatory cells, thus playing an important role in mechanisms involved in secondary brain injury post-TBI. ${ }^{4}$

Complement component $1 \mathrm{q}(\mathrm{C} 1 \mathrm{q})$ is an important component of the classical complement pathway, which is involved in both adaptive and innate immunity via
Correspondence: Guo-Feng Yu Tel +865703055179

Email yuguofeng79@aliyun.com 
activating a cascade of other soluble or membrane bound proteins. ${ }^{5} \mathrm{C} 1 \mathrm{q}$ can be synthesized in microglia and neurons. ${ }^{6,7}$ Its elevated expression has been revealed in brain tissues of animals with acute brain injury and humans with TBI. $^{8,9}$ In patients with acute ischemic stroke, serum C1q levels were significantly increased. ${ }^{10}$ A recent clinical investigation showed that serum $\mathrm{Clq}$ levels were positively associated with severity of neurological impairment and infarct size of patients with acute ischemic stroke. ${ }^{11}$ However, to the best of our knowledge, there is a paucity of data available regarding the relationship between serum C1q levels and the severity plus prognosis of TBI. The purpose of the current study was to determine serum C1q levels after TBI and further evaluate the reliability in regard to serum $\mathrm{Clq}$ for the prediction of long-term poor functional outcome among a cohort of TBI patients.

\section{Materials and Methods}

\section{Design and Subjects}

In this prospective and observational study, we consecutively recruited hospitalized patients with blunt TBI from July 2016 to February 2020. We further excluded those patients who had any one of the following criteria: (1) age less than eighteen years; (2) abbreviated injury scale score of 3 or greater in any non-cranial aspects; (3) admission time exceeding $12 \mathrm{~h}$ since trauma; (4) incomplete information; (5) loss to a 6-month follow-up post- trauma; (6) refusal to participation; (7) unavailable blood sampling and (8) other known severe diseases, eg, malignancy, uremia, liver cirrhosis and heart failure. Alternatively, a group of healthy volunteers were selected as controls. The study was performed in accordance with the tenets of the Declaration of Helsinki. The ethical committee at the Quzhou Affiliated Hospital of Wenzhou Medical University (Quzhou People's Hospital) gave approval for the protocol of this study (opinion number: LW2021-004). Informed consent to participation was signed by participants themselves or their relatives if patients were unconscious.

\section{Recorded Data}

We collected some routine information with respect to demographic data (age and gender), adverse life habits (cigarette smoking and alcohol drinking), coexisting diseases (hypertension, diabetes mellitus and hyperlipidemia), time parameters (admission time and blood-collection time) and traumatic causes (automobile/motorcycle, fall/jump or others). Alcohol abuse was defined as moderate-to severe alcohol consumption in social history $\left(>168 \mathrm{~g} /\right.$ week). ${ }^{12}$ Those patients who smoked regularly at least one cigarette per day at the time of presentation were considered as current smokers. ${ }^{12}$ Systolic and diastolic arterial blood pressures were measured by use of a noninvasive technique. Positive radiological appearances included abnormal cisterns, midline shift, epidural hematoma, subdural hematoma, subarachnoid hemorrhage, intraventricular hemorrhage, intracerebral hematoma, brain contusion and pneumocephalus. Clinical severity was assessed using Glasgow coma scale (GCS) and radiological severity was evaluated utilizing Rotterdam computed tomography (CT) classification. Clinical outcome at post-traumatic 6 months was assessed using 8-grade Extended Glasgow Outcome Scale (GOSE). GOSE score of 1-4 was defined as a poor outcome and GOSE score of 5-8 was regarded as a good outcome. ${ }^{13}$

\section{Detection of Serum Clq}

Venous blood samples were acquired and then centrifuged. Serum was stored at $-80^{\circ} \mathrm{C}$ until assayed. Serum $\mathrm{C} 1 \mathrm{q}$ levels were in duplicate gauged using a commercially available enzyme-linked immunosorbent assay kit in accordance with the manufacturer's instructions (Cloud-Clone Corp., Wuhan, China). Measurements were done in batches by the same technician who was inaccessible to clinical information.

\section{Statistical Analysis}

All data were analyzed using MedCal version 17.9.7. (MedCalc Software, Mariakerke, Belgium) software or the SPSS version 20.0 (SPSS Inc., Chicago, Illinois, USA) statistical package. Variables were reported as mean \pm standard deviation (SD) for normally distributed quantitative data, median (upper-lower quartiles) for nonnormally distributed quantitative data and count (proportion) for qualitative data. The significances of mean and median comparisons between two groups were determined using the Student's $t$-test and Mann-Whitney $U$-test respectively. Qualitative data were compared using the Pearson chi-square test or Fisher's exact test as appropriate. Non-normally quantitative data were compared among multiple groups using the Kruskal-Wallis test. Bivariate correlations were evaluated using Spearman correlation analysis. Simultaneously, the multivariate linear regression analysis was conducted. The multivariable logistic regression analysis was done to identify independent predictors of 6-month poor outcome after head trauma. The associations were reported as odds ratios (ORs) and the corresponding 95\% confidence intervals (CIs). We constructed 
the receiver operating characteristic (ROC) curve to assess the 6-month poor outcome prediction ability of serum $\mathrm{C} 1 \mathrm{q}$ levels. Area under ROC curve (AUC) and its 95\% CI were subsequently generated. Using the maximum Youden $\mathrm{J}$ index, the optimal cut-off value of serum C1q levels was chosen, which yielded the corresponding sensitivity and specificity values for differentiating between patients with a poor outcome and those presenting with a good outcome at 6 months post-injury. $\mathrm{P}<0.05$ was considered to indicate a statistically significant difference.

\section{Results}

\section{Patient Selection and Characteristics}

In the current study, we at first assessed 227 patients with blunt TBI admitted to our hospital. We further excluded 39 patients because of age less than eighteen years (4 cases), abbreviated injury scale score of 3 or greater in any noncranial aspects (8 cases), admission time exceeding 12 $\mathrm{h}$ since trauma (9 cases), incomplete information (3 cases), loss to a 6-month follow-up post- trauma ( 2 cases), refusal to participation ( 1 case), unavailable blood sampling ( 2 cases) and other known severe diseases (10 cases). Ultimately, a total of 188 TBI patients were included in this study. Simultaneously, 188 healthy controls were recruited. There were no substantial differences in age and percentage of sex between controls and patients.

The eventually assessed TBI patients, of whom, 112 were males and 76 were females, were aged from 18 to 78 years (mean, 40.0 years; SD, 14.7 years). Current cigarette smoking and alcohol abuse were found in 48 (25.5\%) and 42 (22.3\%) patients respectively. In total, $31(16.5 \%)$ patients were inflicted with hypertension, $16(8.5 \%)$ coexisted with diabetes mellitus and $32(17.0 \%)$ suffered from hyperlipidemia. Patients were admitted to hospital from 0.5 to 12.0 hours following trauma (median, 5.3 hours; lower-upper quartiles, 2.7-8.3 hours) and peripheral venous blood was obtained from 1.4 to 13.7 hours after trauma (median, 6.8 hours; percentiles $25^{\text {th }}-75^{\text {th }}, 4.7-9.3$ hours). GCS score ranged from 3 to 15 (median, 10; lower-upper quartiles, 7-10). In addition, there were 63 patients with severe TBI (GCS 3-8), 81 with moderate TBI (GCS 9-12) and 44 with mild TBI (GCS 13-15). As regards traumatic causes, almost half of patients had TBI engendered by automobile or motorcycle accidents (92 cases, 48.9\%), other causes of TBI included fall and jump (77 cases, $41.0 \%$ ), and a little portion of patients (19 cases, $10.1 \%$ ) experienced a bicycle mishap, a physical assault, or a sudden collapse. Systolic, diastolic and mean arterial blood pressures ranged from 71 to $177 \mathrm{mmHg}$ (mean, $121.1 \mathrm{mmHg}$; $\mathrm{SD}, 29.8 \mathrm{mmHg}$ ), from 43 to $115 \mathrm{mmHg}$ (mean, $76.3 \mathrm{mmHg}$; $\mathrm{SD}, 20.3 \mathrm{mmHg}$ ) and from 52 to $132 \mathrm{mmHg}$ (mean, 91.3 $\mathrm{mmHg}$; SD, $22.6 \mathrm{mmHg}$ ) respectively. Rotterdam CT classification ranged from 1 to 6 (median, 3; lower-upper quartiles, 2-4). When Rotterdam CT classification was regarded as a categorical variable. There were 25 patients with score 1 , 40 with score 2, 57 with score 3, 34 with score 4, 20 with 5 and 12 with 6 . All patients had essentially cranial injuries, among whom there were 79 patients with abnormal cisterns, 75 showing midline shift above $5 \mathrm{~mm}, 87$ displaying epidural hematoma, 111 exhibiting subdural hematoma, 132 suffering from subarachnoid hemorrhage, 12 presenting with intraventricular hemorrhage, 81 coexisting with intracerebral hematoma, 112 having brain contusion, 53 inflicted with pneumocephalus, 112 with presence of skull-cap fracture and 87 with existence of skull-base fracture. At 6 months after head trauma, GOSE score ranged from 1 to 8 (median, 6; percentiles $\left.25^{\text {th }}-75^{\text {th }}, 4-7\right)$. A total of $52(27.7 \%)$ TBI patients had a poor outcome (GOSE 1-4).

\section{Serum Clq Levels and Severity Plus Inflammation}

In Figure 1, TBI patients had substantially higher serum $\mathrm{C} 1 \mathrm{q}$ levels than controls $(\mathrm{P}<0.001)$. As regards relationship between serum $\mathrm{Clq}$ levels and trauma severity

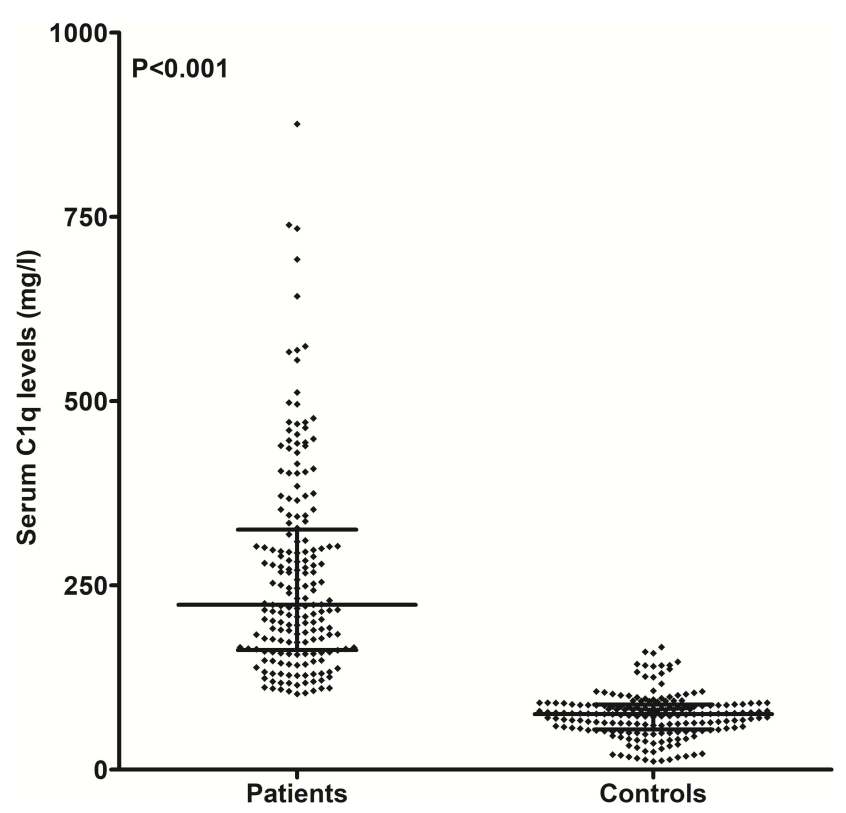

Figure I Differences in terms of serum $\mathrm{Clq}$ levels between healthy controls and patients with traumatic brain injury. Serum $\mathrm{Clq}$ levels were reported as median (upper-lower quartiles). Serum $\mathrm{Clq}$ levels were significantly higher in patients with traumatic brain injury than in healthy controls using the Mann-Whitney U-test. 
indicated by GCS score and Rotterdam CT classification among this group of TBI patients, we found that (1) serum $\mathrm{C} 1 \mathrm{q}$ levels were significantly inversely correlated with GCS score, which was identified as a continuous variable $(\mathrm{P}<0.001)$ (Figure 2); (2) serum $\mathrm{C} 1 \mathrm{q}$ levels were significantly higher in patients with severe TBI (GCS 3-8) than in those experiencing moderate TBI (GCS 9-12) $(\mathrm{P}<0.001)$ and in severe TBI patients than in those

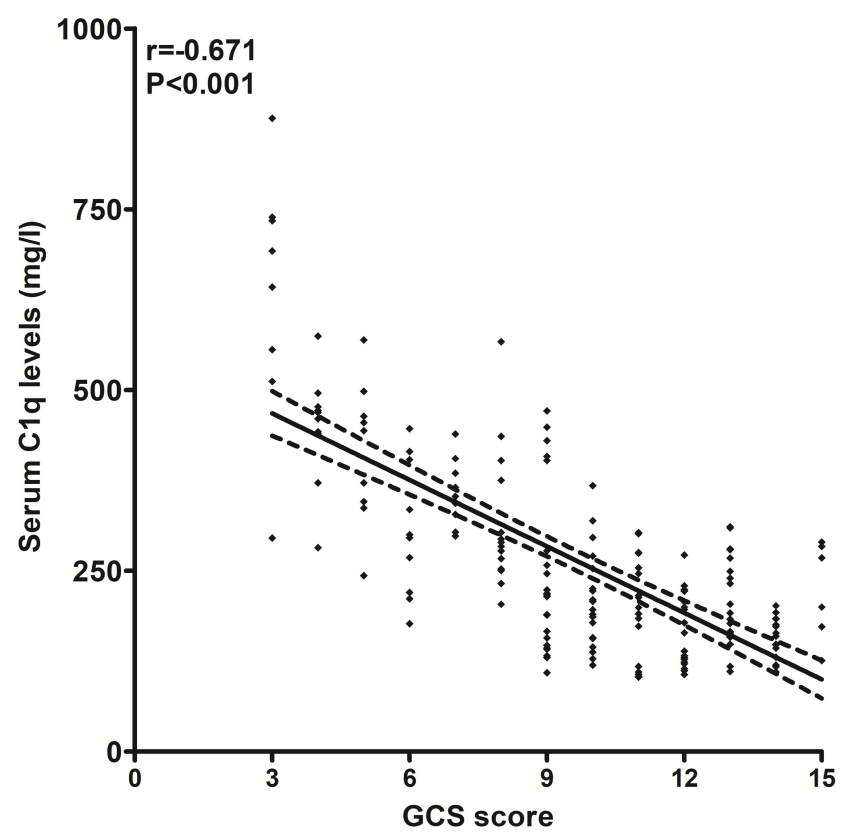

Figure 2 Relationship between serum $\mathrm{Clq}$ levels and Glasgow coma scale score after traumatic brain injury. Serum $\mathrm{Cl} q$ levels were tightly correlated with Glasgow coma scale score following head trauma using the Spearman correlation coefficient. Abbreviation: GCS, Glasgow coma scale. presenting with mild TBI (GCS 13-19) $(\mathrm{P}<0.001)$, while there was no marked difference in terms of serum $\mathrm{Clq}$ levels between patients with moderate TBI and those who presented with mild TBI ( $>0.05)$ (Figure 3); (3) when Rotterdam CT classification was identified as a qualitative variable, there was a significant trend that serum $\mathrm{C} 1 \mathrm{q}$ levels were substantially elevated, with increasing Rotterdam CT classification $(\mathrm{P}<0.001)$ (Figure 4). Alternatively, serum C-reactive protein levels were highly correlated with serum $\mathrm{Clq}$ levels after head trauma $(\mathrm{P}<0.001)$ (Figure 5). A multivariate linear regression model, which contained variables significantly correlated with serum C1q levels in Table 1 (namely, GCS score, Rotterdam CT classification, abnormal cisterns, midline shift above $5 \mathrm{~mm}$, epidural hematoma, subdural hematoma, subarachnoid hemorrhage, intraventricular hemorrhage, intracerebral hematoma, serum $\mathrm{C}$-reactive protein levels and blood glucose levels), showed that serum C1q levels were independently correlated with GCS score ( $\mathrm{t}=$ -3.234, $\mathrm{P}=0.001)$, Rotterdam CT classification $(\mathrm{t}=2.937$, $\mathrm{P}=0.004)$ and serum $\mathrm{C}$-reactive protein levels $(\mathrm{t}=2.589$, $\mathrm{P}=0.010)$.

\section{Serum $\mathrm{Clq}$ Levels and Prognosis}

Figure 6 shows that serum C1q levels were closely and negatively correlated with GOSE score in this host of TBI patients $(\mathrm{P}<0.001)$. Moreover, patients with development of a poor outcome (GOSE 1-4) at 6 months following head trauma had statistically significantly elevated serum C1q levels, as compared with those with a good outcome

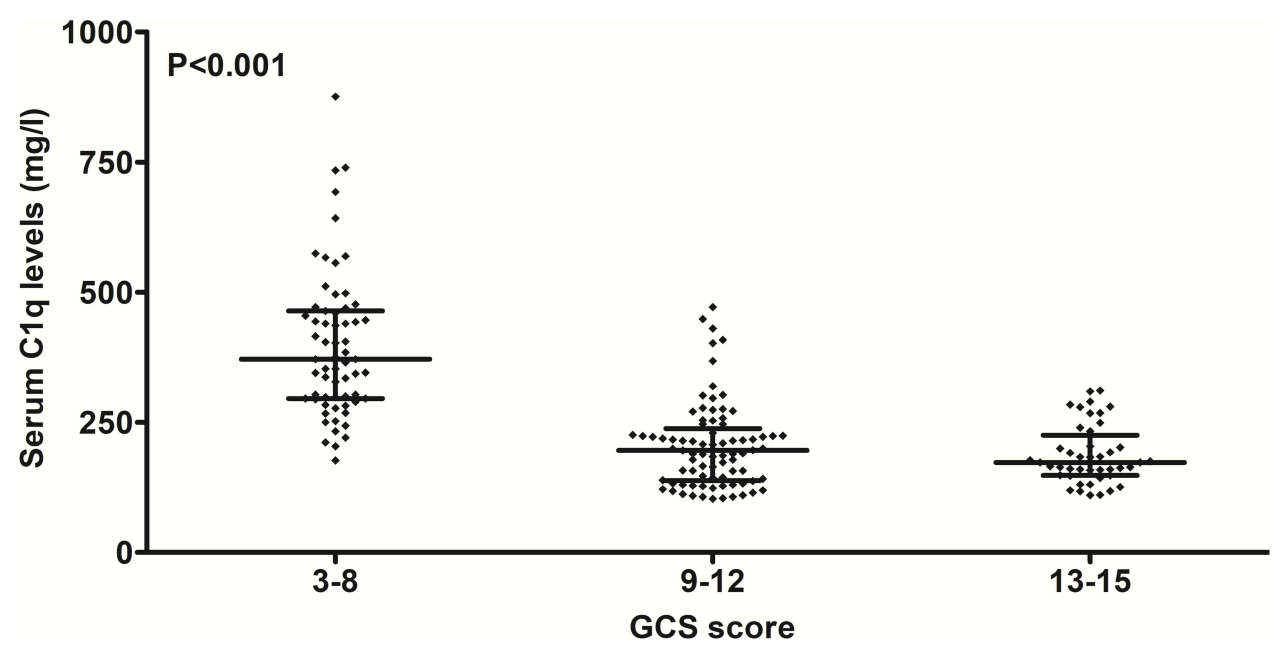

Figure 3 Comparisons of serum Clq levels across severity grade among patients with traumatic brain injury. Significant differences of serum $\mathrm{Cl} q$ levels existed after head trauma among multiple groups using the Kruskal-Wallis test.

Abbreviation: GCS, Glasgow coma scale. 


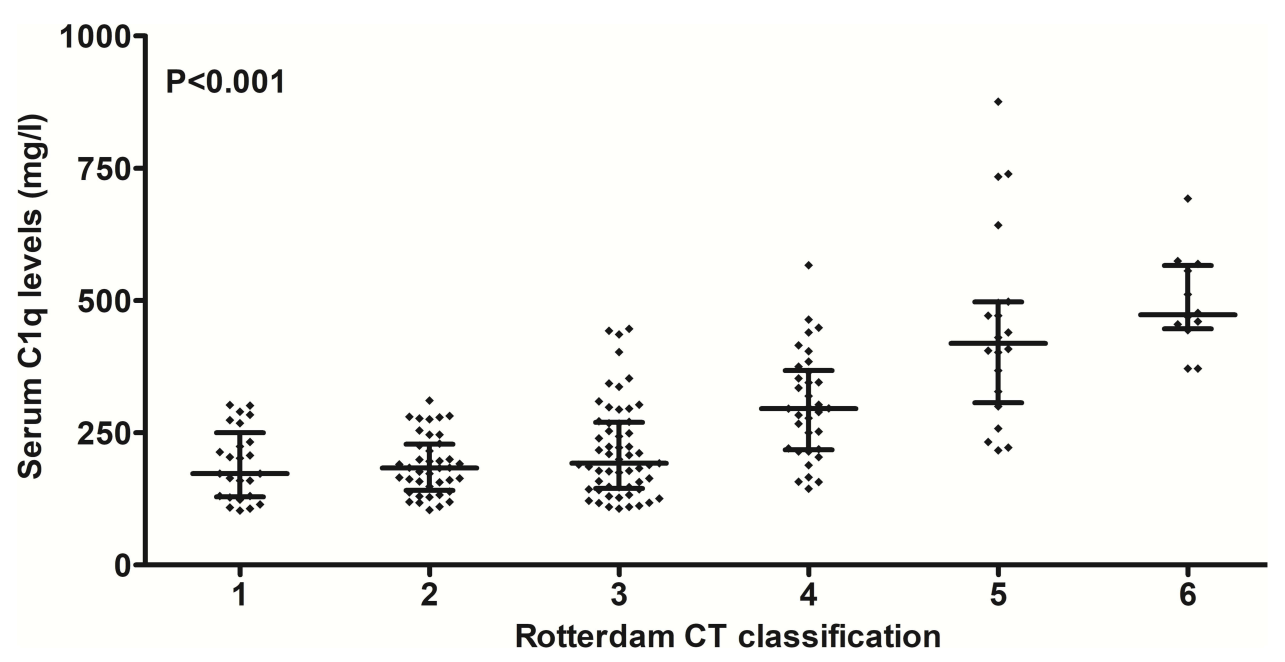

Figure 4 Differences of serum $\mathrm{Clq}$ levels by Rotterdam computerized tomography classification after traumatic brain injury. There were substantial differences in terms of serum $\mathrm{Clq}$ levels after head trauma among multiple groups using the Kruskal-Wallis test. Abbreviation: CT, computerized tomography.

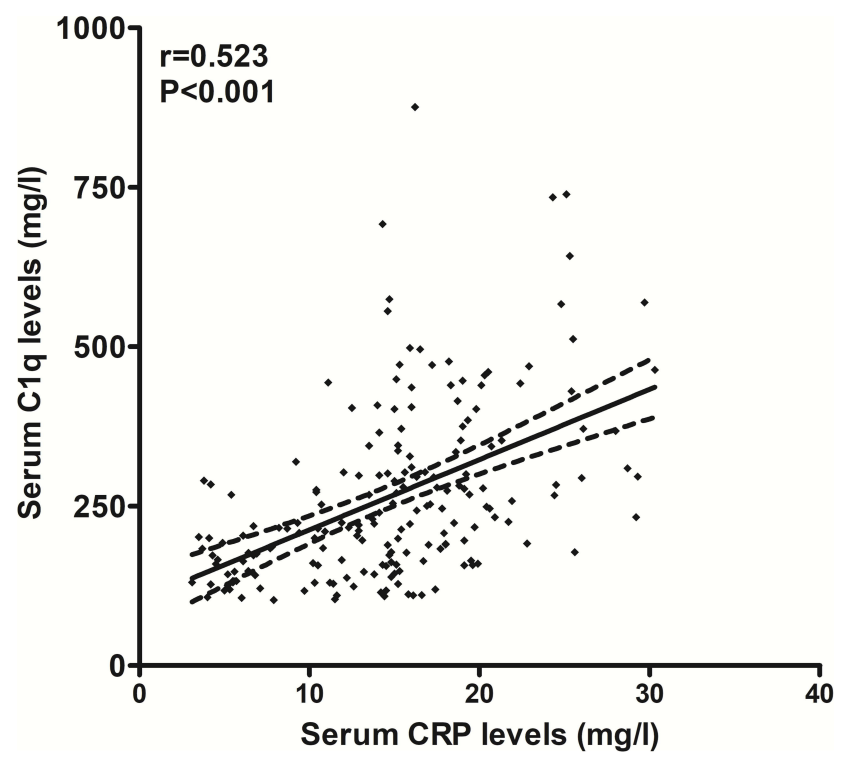

Figure 5 Relation of serum $\mathrm{Clq}$ levels to serum C-reactive protein levels after traumatic brain injury. Serum $\mathrm{Clq}$ levels were closely correlated with serum C-reactive protein levels after head trauma using the Spearman correlation coefficient.

Abbreviation: CRP, C-reactive protein.

$(\mathrm{P}<0.001)$ (Figure 7). Under ROC curve, serum C1q levels distinguished patients with a poor outcome from the other remaining ones with AUC at 0.793 (Table 2). Moreover, using maximum Youden index, an optimal value of serum C1q levels was selected, which was $345.5 \mathrm{mg} / 1$, yielding the corresponding sensitivity and specificity values (Figure 8). In Table 2, the prognostic predictive ability of serum C1q levels was equivalent to those of GCS score and Rotterdam CT classification (both $\mathrm{P}>0.05$ ).
Furthermore, serum C1q levels significantly improved the AUCs of GCS score and Rotterdam CT classification (both $\mathrm{P}<0.05$, Table 2).

In Table 3, using univariate analysis, the associated factors of 6-month poor outcome were as follows: GCS score, Rotterdam CT classification, abnormal cisterns, midline shift more than $5 \mathrm{~mm}$, epidural hematoma, blood glucose levels, serum C-reactive protein levels and serum C1q levels > $345.5 \mathrm{mg} / \mathrm{l}$. The aforementioned significant variables were entered into the multivariate model and as a consequence, the factors retaining independently associated with a poor outcome at post-traumatic 6 months were GCS score (odd ratio, $0.721 ; 95 \%$ CI, 0.591-0.881; $\mathrm{P}=0.001)$, Rotterdam CT classification (odd ratio, 1.849; 95\% CI, 1.247-2.740; $\mathrm{P}=0.007$ ) and serum C1q levels $>345.5 \mathrm{mg} / \mathrm{l}$ (odd ratio, $4.922 ; 95 \% \mathrm{CI}$, $1.552-15.606 ; \mathrm{P}=0.017$ ).

\section{Discussion}

Neuroinflammation happens after TBI. Excessive complement activation is implicated in neuroinflammation and can promote secondary brain injury after $\mathrm{TBI}^{2,4}$ Complement $\mathrm{C} 1 \mathrm{q}$ is identified as a component of the $\mathrm{C} 1$ complex and is involved in both adaptive and innate immunity via the classical complement pathway. ${ }^{3} \mathrm{C} 1 \mathrm{q}$ functional activity has been revealed to be raised markedly in cerebrospinal fluid of rats after transient global cerebral ischemia. ${ }^{8}$ In two previous studies of patients with ischemic stroke, serum C1q levels of patients were substantially elevated as compared to healthy controls or controls without stroke. ${ }^{10,11}$ Our study also displayed 
Table I Univariate Correlation Analysis Between Serum $\mathrm{Clq}$ Levels and Other Variables in 188 Patients with Traumatic Brain Injury

\begin{tabular}{|c|c|c|}
\hline & $\mathbf{r}$ & $P$ value \\
\hline Gender (male/female) & 0.014 & 0.845 \\
\hline Age (y) & 0.118 & 0.107 \\
\hline Current cigarette smoking & -0.064 & 0.384 \\
\hline Alcohol abuse & 0.024 & 0.746 \\
\hline Hypertension & 0.066 & 0.369 \\
\hline Diabetes mellitus & -0.040 & 0.585 \\
\hline Hyperlipidemia & 0.093 & 0.204 \\
\hline Admission time $(\mathrm{h})$ & -0.066 & 0.365 \\
\hline Blood-collection time $(\mathrm{h})$ & -0.045 & 0.543 \\
\hline GCS score & -0.671 & $<0.001$ \\
\hline Traumatic causes & -0.074 & 0.310 \\
\hline Systolic arterial pressure $(\mathrm{mmHg})$ & 0.059 & 0.424 \\
\hline Diastolic arterial pressure $(\mathrm{mmH})$ & 0.097 & 0.185 \\
\hline Mean arterial pressure $(\mathrm{mmHg})$ & 0.091 & 0.213 \\
\hline Rotterdam CT classification & 0.604 & $<0.00$ I \\
\hline Abnormal cisterns & 0.725 & $<0.00$ I \\
\hline Midline shift $>5 \mathrm{~mm}$ & 0.457 & $<0.001$ \\
\hline Epidural hematoma & 0.246 & 0.001 \\
\hline Subdural hematoma & 0.199 & 0.006 \\
\hline Subarachnoid hemorrhage & 0.168 & 0.021 \\
\hline Intraventricular hemorrhage & 0.251 & 0.001 \\
\hline Intracerebral hematoma & 0.234 & 0.001 \\
\hline Brain contusion & 0.130 & 0.075 \\
\hline Pneumocephalus & 0.057 & $0.44 I$ \\
\hline Skull-cap fracture & 0.031 & 0.677 \\
\hline Skull-base fracture & -0.011 & 0.879 \\
\hline Serum CRP levels (mg/l) & 0.523 & $<0.001$ \\
\hline Blood glucose levels (mmol/l) & 0.262 & $<0.00$ I \\
\hline Blood leucocyte count $\left(\times 10^{9}\right)$ & 0.112 & 0.126 \\
\hline
\end{tabular}

Note: Results were reported as $r$ values using Spearman correlation analysis. Abbreviations: GCS, Glasgow coma scale; CT, computerized tomography; CRP, C-reactive protein.

that serum $\mathrm{Clq}$ levels were statistically significantly enhanced in patients with TBI than in healthy controls. Moreover, C1q expressions were significantly increased in rat brain microglia following the ischemic insult. ${ }^{14}$ A dramatical up-regulation of C1q expression was confirmed in cortex, striatum, thalamus and hippocampus of mice exposed to controlled cortical impact, as well as it was localized in microglia and neurons. ${ }^{15}$ Interestingly, in patients with TBI, elevated levels of C1q were found in the penumbra of the injured area. ${ }^{9}$ Thus, it is presumed that $\mathrm{Clq}$ in serum of TBI patients might be at least partially derived from injured brain tissues.

$\mathrm{C} 1 \mathrm{q}$ possesses various complement and noncomplement functions. ${ }^{5} \mathrm{C} 1 \mathrm{q}$ may participate in synaptic pruning, thereby exerting a key role in shaping neuronal

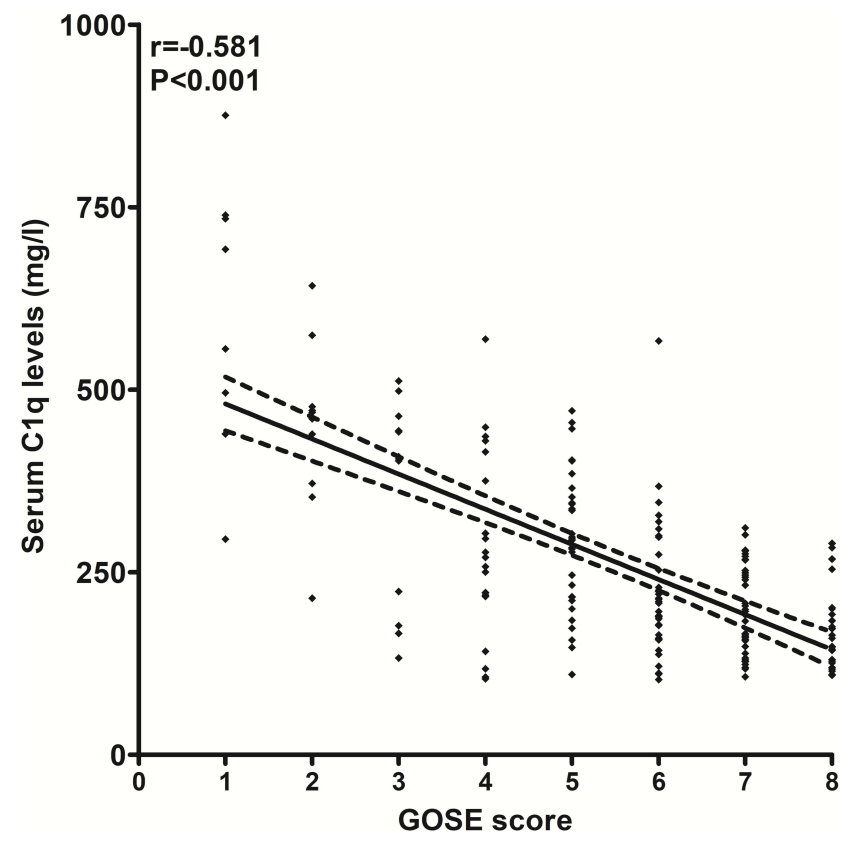

Figure 6 Association of serum $\mathrm{Clq}$ levels with extended Glasgow outcome scale score after head trauma. Serum $\mathrm{Clq}$ levels were highly correlated with extended Glasgow outcome scale score after head trauma using the Spearman correlation coefficient. Abbreviation: GOSE, Glasgow outcome scale score.

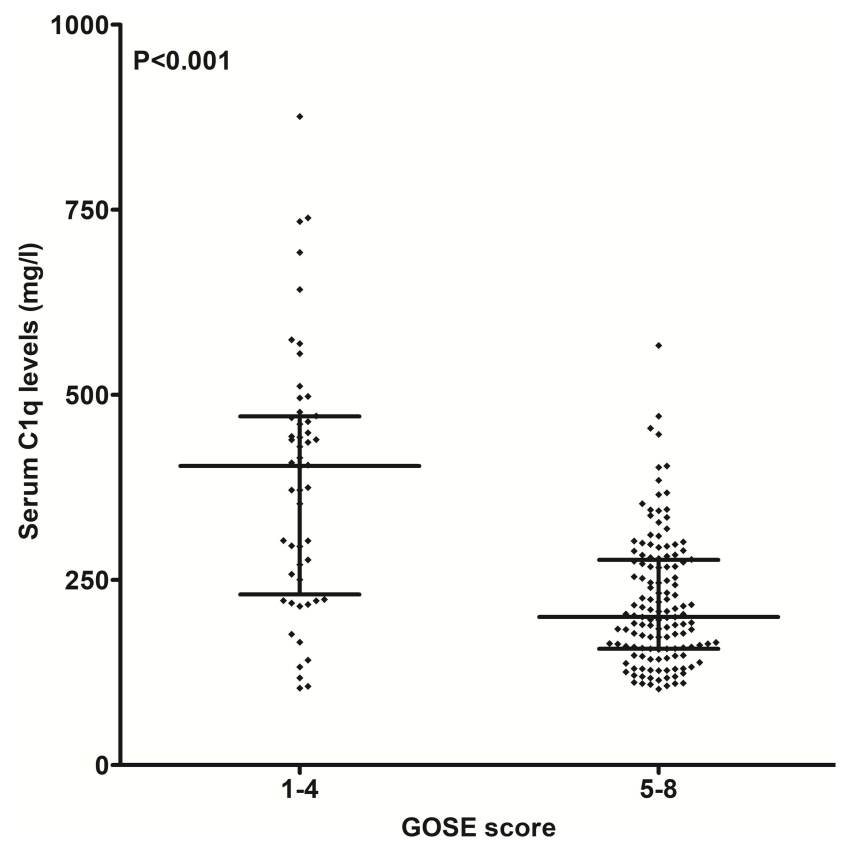

Figure 7 Differences of serum $\mathrm{Clq}$ levels between patients with extended Glasgow outcome scale score $1-4$ and those with extended Glasgow outcome scale score 5-8 at 6 months after traumatic brain injury. Serum $\mathrm{Clq}$ levels were markedly raised in patients with extended Glasgow outcome scale score 1-4, as compared to those presenting with score 5-8 at 6 months after head trauma using the Mann-Whitney U-test.

Abbreviation: GOSE, Glasgow outcome scale score.

architecture during the development of the central nervous system. ${ }^{16,17}$ Also, C1q was demonstrated to enhance microglial clearance of apoptotic cells using primary rat 
Table 2 Receiver Operating Characteristic Curve Analysis for Predicting 6-Month Poor Outcome After Traumatic Brain Injury

\begin{tabular}{|l|c|c|c|c|}
\hline & AUC (95\% Cl) & P value & P value & P value \\
\hline Serum Clq levels (mg/l) & $0.793(0.728-0.849)$ & Reference & \\
GCS score & $0.844(0.784-0.893)$ & 0.1084 & Reference \\
Rotterdam CT classification & $0.806(0.742-0.860)$ & 0.6922 & \multirow{2}{*}{ Reference } \\
Combined GCS score and serum Clq levels & $0.866(0.809-0.911)$ & & 0.0403 & \\
Combined Rotterdam CT classification and serum Clq levels & $0.853(0.794-0.900)$ & & 0.0324 \\
\hline
\end{tabular}

Notes: A poor outcome was defined as extended Glasgow outcome scale score of I-4 at 6 months after head trauma. In a combined Logistic regression model, additive effect of a variable to another one was assessed. $Z$ test was performed to compare areas under curve between two variables.

Abbreviations: GCS, Glasgow coma scale; TBI, traumatic brain injury; CT, computerized tomography; ROC, receiver operating characteristic; AUC, area under curve; GOSE, Extended Glasgow Outcome Scale; RAGE, advanced glycation end-product; OR, odds ratio; $95 \% \mathrm{Cl}$, 95\% confidence interval; AUC, area under curve; $95 \% \mathrm{Cl}$, 95\% confidence interval; CT, computed tomography; GCS, Glasgow coma scale.

microglia and neurons. ${ }^{18}$ In addition, in cultured microglial cells, extrinsic C1q markedly increased intracellular $\mathrm{Ca}^{2+}$ levels and the release of interleukin-6, tumor necrosis factor-alpha and nitric oxide. ${ }^{19}$ In another study, C1q suppressed the lipopolysaccharide -induced production of proinflammatory cytokines (such as interleukin-1 alpha, interleukin-1beta, interleukin-6 and tumor necrosis factoralpha) from cultured microglial cells, while the presence of $\mathrm{Clq}$ enhanced levels of monocyte chemoattractant protein- $1 .{ }^{18}$ In line with such data, our study found that serum C1q levels were strongly correlated with serum C-reactive protein levels after TBI. Receptor for advanced glycation end-product (RAGE) is related to the brain proinflammatory signaling pathways and is also a receptor for C1q. $^{20}$ Thus, C1q/RAGE complexes may regulate

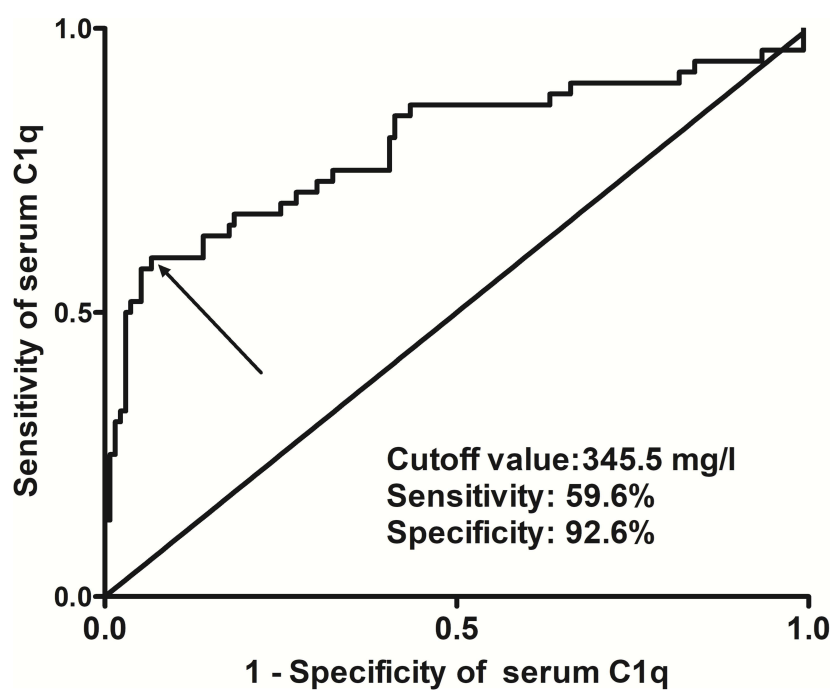

Figure 8 Analysis regarding the predictive ability of serum $\mathrm{Clq}$ levels for 6-month poor outcome after traumatic brain injury. Poor outcome was defined as extended Glasgow outcome scale score 1-4. Discriminatory capability was assessed under receiver operating characteristic curve. Using maximum Youden index, an optimal value of serum $\mathrm{Clq}$ levels was selected. Arrow indicates the selected cutoff value of serum Clq levels, namely, $345.5 \mathrm{mg} / \mathrm{l}$, which predicted 6-month poor outcome with $59.6 \%$ sensitivity and $92.6 \%$ specificity. neuroinflammation. The preceding evidence is supportive of the notion that $\mathrm{C} 1 \mathrm{q}$ might regulate neuroinflammation responding to acute brain injury. In mice with middle cerebral artery occlusion, the complement inhibitor soluble complement receptor-1 significantly lessened cerebral infarct volume and improved neurologic outcome, presumably in large part through the blockade of the downstream effects of postischemic cerebral C1q expression. ${ }^{21}$ Another experiment showed that C1q-deficiency may be neuroprotective against hypoxic-ischemic brain injury in neonatal mice. $^{22}$ Thus, C1q may emerge as a therapeutic target for preventing inflammatory injury after acute brain injury.

To the best of our knowledge, no data have existed regarding relationship between serum $\mathrm{C} 1 \mathrm{q}$ levels and severity plus prognosis after TBI. However, there are two human studies demonstrating its close association with severity and prognosis after ischemic stroke. ${ }^{10,11}$ In a study of patients with acute ischemic stroke, serum C1q levels were substantially higher in patients than in healthy controls and its elevated levels were significantly positively correlated with infarction volumes. ${ }^{10}$ In another study, serum C1q levels were positively associated with severity of neurological impairment and infarct size of patients with acute ischemic stroke. ${ }^{11}$ Nevertheless, a long-term neurologic function assessment was not done in those two studies of humans with acute ischemic stroke. ${ }^{10,11}$ Our study not only found that elevated serum C1q levels, as compared to healthy controls, were markedly correlated with trauma severity indicated by GCS and Rotterdam CT classification, but also showed that serum C1q levels were independently associated with 6-month poor outcome. More intriguingly, serum Clq levels displayed similar prognostic capabilities, as compared to GCS scores and Rotterdam CT classification, as well as dramatically improved prognostic abilities of GCS scores and Rotterdam CT classification based on ROC curve. In 
Table 3 Differences in Baseline Characteristics by a Poor Outcome at 6 Months After Traumatic Brain Injury

\begin{tabular}{|c|c|c|c|}
\hline & GOSE I-4 & GOSE 5-8 & $P$ value \\
\hline Number & 52 & 136 & \\
\hline Gender (male/female) & $27 / 25$ & $85 / 51$ & 0.186 \\
\hline Age $(y)$ & $42.4 \pm 14.8$ & $39.0 \pm 14.6$ & 0.164 \\
\hline Current cigarette smoking & $14(26.9 \%)$ & $34(25.0 \%)$ & 0.787 \\
\hline Alcohol abuse & $10(19.2 \%)$ & $32(23.5 \%)$ & 0.527 \\
\hline Hypertension & $\mathrm{II}(2 \mathrm{I} .2 \%)$ & $20(14.7 \%)$ & 0.287 \\
\hline Diabetes mellitus & $5(9.6 \%)$ & II (8.1\%) & 0.965 \\
\hline Hyperlipidemia & $12(23.1 \%)$ & $20(14.7 \%)$ & 0.172 \\
\hline Admission time $(\mathrm{h})$ & $5.2(2.3-9.5)$ & $5.4(3.0-8.0)$ & 0.880 \\
\hline Blood-collection time $(\mathrm{h})$ & $6.8(4.4-11.0)$ & $6.9(4.7-9.2)$ & 0.920 \\
\hline GCS score & $7(4-9)$ & II (9-13) & $<0.001$ \\
\hline Traumatic causes & & & 0.734 \\
\hline Automobile/motorcycle & 27 & 65 & \\
\hline Fall/jump & 19 & 58 & \\
\hline Others & 6 & 13 & \\
\hline Systolic arterial pressure $(\mathrm{mmHg})$ & $117.4 \pm 33.7$ & $122.5 \pm 28.2$ & 0.293 \\
\hline Diastolic arterial pressure $(\mathrm{mmHg})$ & $73.8 \pm 21.7$ & $77.3 \pm 19.7$ & 0.288 \\
\hline Mean arterial pressure $(\mathrm{mmHg})$ & $88.3 \pm 25.2$ & $92.4 \pm 21.6$ & 0.274 \\
\hline Rotterdam CT classification & $4(3-5)$ & $3(2-3)$ & $<0.001$ \\
\hline Abnormal cisterns & $43(82.3 \%)$ & $36(26.5 \%)$ & $<0.001$ \\
\hline Midline shift $>5 \mathrm{~mm}$ & $36(69.2 \%)$ & $39(28.7 \%)$ & $<0.001$ \\
\hline Epidural hematoma & $3 \mathrm{I}(59.6 \%)$ & $56(4 I .2 \%)$ & 0.023 \\
\hline Subdural hematoma & $32(61.5 \%)$ & $79(58.1 \%)$ & 0.667 \\
\hline Subarachnoid hemorrhage & $39(75.0 \%)$ & $93(68.4 \%)$ & 0.375 \\
\hline Intraventricular hemorrhage & $6(11.5 \%)$ & $6(4.4 \%)$ & 0.095 \\
\hline Intracerebral hematoma & $25(48.1 \%)$ & $56(4 I .2 \%)$ & 0.393 \\
\hline Brain contusion & $34(65.4 \%)$ & 78 (57.4\%) & 0.315 \\
\hline Pneumocephalus & $20(38.5 \%)$ & $33(24.3 \%)$ & 0.053 \\
\hline Skull-cap fracture & $33(63.5 \%)$ & $79(58.1 \%)$ & 0.502 \\
\hline Skull-base fracture & $26(50.0 \%)$ & $6 \mathrm{I}(44.9 \%)$ & 0.527 \\
\hline Serum CRP levels (mg/l) & I6.I (14.1-20.3) & $14.5(10.3-17.9)$ & 0.004 \\
\hline Blood glucose levels (mmol/l) & $12.4(8.7-14.7)$ & II.I (6.7-13.3) & 0.036 \\
\hline Blood leucocyte count $\left(\times 10^{9}\right)$ & $8.9(6.3-12.1)$ & $8.7(6.8-11.3)$ & 0.809 \\
\hline Serum Clq levels $>345.5 \mathrm{mg} / \mathrm{l}$ & $31(59.6 \%)$ & $10(7.4 \%)$ & $<0.001$ \\
\hline
\end{tabular}

Notes: Variables were shown as mean \pm standard deviation, median (25th-75th percentiles) or counts (percentages) where appropriate. Comparisons were done using the $t$-tests, Mann-Whitney U-tests, Pearson chi-square test or Fisher's exact test as appropriate.

Abbreviations: GCS, Glasgow coma scale; CT, computerized tomography; CRP, C-reactive protein.

summary, serum C1q may be a potential prognostic biomarker of TBI.

There is at least a limitation to mention in the current study. This study recruited two groups of subjects, namely, healthy controls and patients with traumatic brain injury. Although there were no significant intergroup differences in terms of age and percentage of sex, other confounding factors were not investigated. Thus, the change of serum C1q levels between healthy controls and patients with traumatic brain injury may need to be further validated.

\section{Conclusions}

To the best of our knowledge, our study, for the first time, found that elevated serum $\mathrm{Clq}$ levels, in close correlation with GCS score and Rotterdam CT classification, are independently associated with 6-month poor prognosis after TBI and significantly improve prognostic abilities of GCS score and Rotterdam CT classification among such a group of TBI patients, substantializing serum $\mathrm{C} 1 \mathrm{q}$ as a useful prognostic predictor for brain trauma. 


\section{Abbreviations}

GCS, Glasgow coma scale; TBI, traumatic brain injury; $\mathrm{CT}$, computerized tomography; ROC, receiver operating characteristic; AUC, area under curve; GOSE, Extended Glasgow Outcome Scale; RAGE, advanced glycation endproduct; OR, odds ratio; $95 \% \mathrm{CI}, 95 \%$ confidence interval.

\section{Acknowledgments}

The authors thank all staffs in Department of Neurosurgery, The Quzhou Affiliated Hospital of Wenzhou Medical University, Quzhou People's Hospital (Quzhou, China) for their technical support.

\section{Disclosure}

The authors declare that there were no conflicts of interest.

\section{References}

1. Khellaf A, Khan DZ, Helmy A. Recent advances in traumatic brain injury. J Neurol. 2019;266(11):2878-2889. doi:10.1007/s00415-01909541-4

2. Killen MJ, Giorgi-Coll S, Helmy A, Hutchinson PJ, Carpenter KL. Metabolism and inflammation: implications for traumatic brain injury therapeutics. Expert Rev Neurother. 2019;19(3):227-242. doi:10.1080/ 14737175.2019.1582332

3. Dalakas MC, Alexopoulos H, Spaeth PJ. Complement in neurological disorders and emerging complement-targeted therapeutics. Nat Rev Neurol. 2020;16(11):601-617. doi:10.1038/s41582-020-0400-0

4. Roselli F, Karasu E, Volpe C, Huber-Lang M. Medusa's head: the complement system in traumatic brain and spinal cord injury. J Neurotrauma. 2018;35(2):226-240. doi:10.1089/neu.2017.5168

5. Kishore U, Reid KB. C1q: structure, function, and receptors. Immunopharmacology. 2000;49(1-2):159-170. doi:10.1016/s01623109(00)80301-x

6. Fonseca MI, Chu SH, Hernandez MX, et al. Cell-specific deletion of C1qa identifies microglia as the dominant source of $\mathrm{C} 1 \mathrm{q}$ in mouse brain. J Neuroinflammation. 2017;14(1):48. doi:10.1186/s12974-017-0814-9

7. Datta D, Leslie SN, Morozov YM, et al. Classical complement cascade initiating $\mathrm{C} 1 \mathrm{q}$ protein within neurons in the aged rhesus macaque dorsolateral prefrontal cortex. J Neuroinflammation. 2020;17(1):8. doi:10.1186/s12974-019-1683-1

8. Schäfer MK, Schwaeble WJ, Post C, et al. Complement C1q is dramatically up-regulated in brain microglia in response to transient global cerebral ischemia. $J$ Immunol. 2000;164(10):5446-5452. doi:10.4049/jimmunol.164.10.5446

9. Bellander BM, Singhrao SK, Ohlsson M, Mattsson P, Svensson M. Complement activation in the human brain after traumatic head injury $J \quad$ Neurotrauma. 2001;18(12):1295-1311. doi:10.1089/0897 7150152725605

Neuropsychiatric Disease and Treatment

\section{Publish your work in this journal}

Neuropsychiatric Disease and Treatment is an international, peerreviewed journal of clinical therapeutics and pharmacology focusing on concise rapid reporting of clinical or pre-clinical studies on a range of neuropsychiatric and neurological disorders. This journal is indexed on PubMed Central, the 'PsycINFO' database and CAS, and
10. Zhao X, Wang C, Pang B, Zhu Y, Zhang Y. The value of serum complement $\mathrm{C} 1 \mathrm{q}$ in the diagnosis of acute ischemic stroke. Clin Lab. 2017;63(5):915-920. doi:10.7754/Clin.Lab.2016.161033

11. Wang DD, Hou XH, Li HQ, et al. Association of serum complement C1q concentration with severity of neurological impairment and infarct size in patients with acute ischemic stroke. J Stroke Cerebrovasc Dis. 2020;29(12):105363. doi:10.1016/j. jstrokecerebrovasdis.2020.105363

12. Kim BS, Jung HS, Bang OY, Chung CS, Lee KH, Kim GM. Elevated serum lipoprotein(a) as a potential predictor for combined intracranial and extracranial artery stenosis in patients with ischemic stroke. Atherosclerosis. 2010;212(2):682-688. doi:10.1016/j. atherosclerosis.2010.07.007

13. Stein DM, Hu PF, Brenner M, et al. Brief episodes of intracranial hypertension and cerebral hypoperfusion are associated with poor functional outcome after severe traumatic brain injury. $J$ Trauma. 2011;71(2):364-373;discussion 373-374. doi:10.1097/ TA.0b013e31822820da

14. Mack WJ, Sughrue ME, Ducruet AF, et al. Temporal pattern of C1q deposition after transient focal cerebral ischemia. $J$ Neurosci Res. 2006;83(5):883-889. doi:10.1002/jnr.20775

15. Ciechanowska A, Ciapała K, Pawlik K, et al. Initiators of classical and lectin complement pathways are differently engaged after traumatic brain injury-time-dependent changes in the cortex, striatum, thalamus and hippocampus in a mouse model. Int J Mol Sci. 2020;22 (1):45. doi:10.3390/ijms22010045

16. Stevens B, Allen NJ, Vazquez LE, et al. The classical complement cascade mediates CNS synapse elimination. Cell. 2007;131 (6):1164-1178. doi:10.1016/j.cell.2007.10.036

17. Stephan AH, Barres BA, Stevens B. The complement system: an unexpected role in synaptic pruning during development and disease. Annu Rev Neurosci. 2012;35:369-389. doi:10.1146/annurev-neuro -061010-113810

18. Fraser DA, Pisalyaput K, Tenner AJ. C1q enhances microglial clearance of apoptotic neurons and neuronal blebs, and modulates subsequent inflammatory cytokine production. J Neurochem. 2010;112 (3):733-743. doi:10.1111/j.1471-4159.2009.06494.x

19. Färber K, Cheung G, Mitchell D, et al. C1q, the recognition subcomponent of the classical pathway of complement, drives microglial activation. J Neurosci Res. 2009;87(3):644-652. doi:10.1002/ jnr.21875

20. Ma W, Rai V, Hudson BI, Song F, Schmidt AM, Barile GR. RAGE binds $\mathrm{C} 1 \mathrm{q}$ and enhances $\mathrm{C} 1 \mathrm{q}-$-mediated phagocytosis. Cell Immunol. 2012;274(1-2):72-82. doi:10.1016/j.cellimm.2012.02.001S00088749(12)00022-6.

21. Huang J, Kim LJ, Mealey R, et al. Neuronal protection in stroke by an sLex-glycosylated complement inhibitory protein. Science. 1999;285(5427):595-599. doi:10.1126/science.285.5427.595

22. Ten VS, Sosunov SA, Mazer SP, et al. C1q-deficiency is neuroprotective against hypoxic-ischemic brain injury in neonatal mice. Stroke. 2005;36(10):2244-2250. doi:10.1161/01.STR.000018 2237.20807.d0 is the official journal of The International Neuropsychiatric Association (INA). The manuscript management system is completely online and includes a very quick and fair peer-review system, which is all easy to use. Visit http://www.dovepress.com/testimonials.php to read real quotes from published authors. 